Notre Dame Journal of Formal Logic

Volume VII, Number 1, January 1966

\title{
ON A PROPER CLASS AND RELATED MATTERS
}

\author{
ALBERT A. MULLIN
}

This note establishes that a subdirect product of certain semigroups (abelian semigroups, monoids, abelian monoids, groups, abelian groups, rings and logical theories [1]) does not exist and a fortiori that certain classes of logico-algebraic structures are proper classes (i.e., classes which are not sets) in the sense of K. Gödel's set theory [2].

Consider an aggregate $\left\{S_{i}\right\}$ of all pair-wise inequivalent semigroups $S_{i}$. If $\left\{S_{i}\right\}$ is a set then its ordinary direct product $P$ (alias, subdirect product; alias complete direct product) exists $\dot{a} l a$ category theory. If $P$ exists then, trivially, $P$ is a semigroup. In such a case $P$ is not inequivalent to every $S_{i}$ for the following reason. If $P$ is inequivalent to every $S_{i}$ then, surely, $P \notin\left\{S_{i}\right\}$ for, otherwise, $P \cong P$; a contradiction of inequivalence. But, then, $\left\{S_{i}\right\}$ does not contain all pair-wise inequivalent semigroups, viz., it does not contain $P$; but this contradicts the definition of $\left\{S_{i}\right\}$ as the class of all pairwise inequivalent semigroups. Hence $P \cong S_{j}$ for some $j$. However if $P \cong S_{j}$ and $P \cong S_{k}, j \neq k$, then $S_{j} \cong S_{k}$; a contradiction of pair-wise inequivalence. Hence $P \cong S_{j}$ for precisely one $j$. Let $S_{j}$ be respesented as $\left(S_{j}{ }^{\prime}, o\right)$ and let $P$ be represented as $\left(P^{\prime}, *\right)$ where ' $O$ ' and ' $*$ ' denote associative composition laws. Since $S_{j}{ }^{\prime}$ is a set one can form its power set $\Pi$, [2]. But there is a semigroup model for every infinite cardinal. Hence there is an $S_{k}$ of the form $(\Pi, \oplus)$ for some $k$. However since $P \cong S_{j}$, card $\left(P^{\prime}\right)=\operatorname{card}\left(S_{j}{ }^{\prime}\right)$ and, therefore, card $(\Pi)>$ card $\left(S_{j}^{\prime}\right)=$ card $\left(P^{\prime}\right)$. But this contradicts the theorem which asserts that the cardinality of a cartesian product of nonempty sets is greater than or equal to the cardinality of any one of its factors. Thus we arrive at the Metatheorem : $P$ does not exist and $\left\{S_{i}\right\}$ is not a set in Gödel's set theory but rather it is a proper class [2]. Metacorollary: The class of all semigroups is not a set, for otherwise, upon forming its power set it would follow that $\left\{S_{i}\right\}$ is a set; a contradiction to the metatheorem.

The author acknowledges the help of colleagues in polishing the results and the assistance of the U.S. NATIONAL SCIENCE FOUNDATION for financial aid. 


\section{REFERENCES}

[1] A. Mostowski, On direct products of theories, The Journal of Symbolic Logic, vol. 17 (1952), pp. 1-31.

[2] K. G8del, Consistency of the Continum Hypothesis, Princeton, 1940.

Lawrence Radiation Laboratory, University of California

Livermore, California 